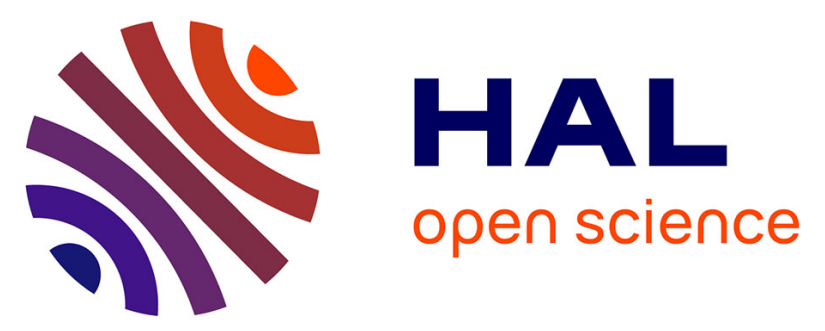

\title{
Increased tolerance to organic xenobiotics following recent allopolyploidy in Spartina (Poaceae)
}

Armand Cavé-Radet, Armel Salmon, Oscar Lima, Malika Ainouche, Abdelhak El Amrani

\section{- To cite this version:}

Armand Cavé-Radet, Armel Salmon, Oscar Lima, Malika Ainouche, Abdelhak El Amrani. Increased tolerance to organic xenobiotics following recent allopolyploidy in Spartina (Poaceae). Plant Science, 2019, 280, pp.143-154. 10.1016/j.plantsci.2018.11.005 . hal-01954014

HAL Id: hal-01954014 https://hal-univ-rennes1.archives-ouvertes.fr/hal-01954014

Submitted on 17 Jan 2019

HAL is a multi-disciplinary open access archive for the deposit and dissemination of scientific research documents, whether they are published or not. The documents may come from teaching and research institutions in France or abroad, or from public or private research centers.
L'archive ouverte pluridisciplinaire HAL, est destinée au dépôt et à la diffusion de documents scientifiques de niveau recherche, publiés ou non, émanant des établissements d'enseignement et de recherche français ou étrangers, des laboratoires publics ou privés. 
Title: Increased tolerance to organic xenobiotics following recent allopolyploidy in Spartina (Poaceae)

Authors: Armand Cavé-Radet ${ }^{1}$, Armel Salmon ${ }^{1}$, Oscar Lima ${ }^{1}$, Malika L. Ainouche ${ }^{1}$ and Abdelhak El Amrani ${ }^{1} \dagger$

${ }^{1}$ Université de Rennes 1, OSUR/CNRS-UMR 6553, Ecosystèmes-Biodiversité-Evolution, Campus de Beaulieu, Bâtiment 14A, 35042 Rennes cedex, France.

$\dagger$ Corresponding author: Abdelhak El Amrani (Email: abdelhak.elamrani@univ-rennes1.fr)

Université de Rennes 1

Centre National de la Recherche Scientifique

UMR 6553 Ecosystems-Biodiversity-Evolution

Campus de Beaulieu, Bâtiment 14A

263 avenue du Général Leclerc

F-35042 Rennes Cedex, France

\section{Highlights:}

- First PAH tolerance comparative analyses in Spartina.

- Increased tolerance highlighted in the allopolyploid $S$. anglica compared to its parents.

- Expression analysis of candidate GST genes (Tau family related) in Spartina.

- Allopolyploidization and phenanthrene induced stress reprogrammed GST gene expression patterns.

- The neoallopolyploid S. anglica is a suitable candidate for phytoremediation.

\section{Abstract}

Genome doubling or polyploidy is a widespread phenomenon in plants where it has important evolutionary consequences affecting the species distribution and ecology. PAHs are ubiquitous organic pollutants, which represent a major environmental concern. Recent data showed that tolerance to organic xenobiotics involve specific signaling pathways, and detoxifying gene sets referred as 'the xenome'. However, no data are available about how polyploidy impacts tolerance to organic xenobiotics. In the present paper, we investigated PAH tolerance following allopolyploidization in Spartina alterniflora, S. maritima and their derived allopolyploid species $S$. anglica. We performed comparative analyses of cellular compartmentalization, photosynthetic indices, and oxidative stress markers under phenanthrene-induced stress, and found that $S$. anglica 
exhibit increased tolerance compared to its parents. Based on 52 genes potentially involved in phenanthrene detoxification previously identified in A. thaliana, we investigated the Spartina xenome using genomic and transcriptomic available resources. Subsequently, we focused on GSTs, a ubiquitous enzymes class involved in organic xenobiotic detoxification. We examined expression profiles of selected genes by RT-qPCR, and revealed various patterns of parental expression alteration in the allopolyploid. The impacts of allopolyploidization on phenanthreneinduced stress and their potential ecological implications are discussed. The neo-allopolyploid $S$. anglica appears as a potential candidate for phytoremediation in PAH-polluted marshes.

Key words: Genome doubling, PAHs, abiotic stress, GSTs, Spartina

\section{INTRODUCTION}

Whole genome duplication (WGD, or polyploidization) is an important mechanism in plant evolutionary history [1]. Polyploidy is accompanied by significant structural and functional genome reorganizations, which may affect the adaptive dynamics of newly formed species (e.g. larger habitats, increased fitness) [2-5]. In allopolyploids, both genome merger ("genomic shock" resulting from interspecific hybridization) and genome redundancy (resulting from genome doubling per se) result in novel genetic and epigenetic interactions, affecting the subsequent evolution and adaptation of species [6].

In this paper, we explore the hypothesis of increased stress tolerance to organic xenobiotics following allopolyploidy in Spartina species. Spartina is a monophyletic lineage that was recently included in the former paraphyletic genus Sporobolus [7]. This lineage is affected by recurrent hybridization and genome duplication, and includes 13 to 15 perennial species colonizing estuaries, coastlines and continental wetlands worldwide. Three species are of particular ecological and evolutionary interests: the European native $S$. maritima (Curtis) Fernald $(2 \mathrm{n}=6 \mathrm{x}=$ $60)$, the recently introduced to Europe from eastern America $S$. alterniflora Loisel. $(2 n=6 x=62)$, and the allopolyploid $S$. anglica C. E. Hubbard $(2 n=12 x=120,122$ or 124). Spartina alterniflora (maternal parent) hybridized with $S$. maritima (paternal parent) which resulted in the formation of a sterile F1 hybrid S. $x$ townsendii, discovered in 1870 (H. Groves \& J. Groves; 2n =6x =62). A few decades later (around the 1890's), chromosome doubling of this hybrid led to the formation of a new fertile and vigorous allododecaploid species S. anglica (see Supplemental Fig. S1), now being listed among the 100 worst invasive alien species (IUCN list [8]). Spartina anglica represents a classic example of recent allopolyploid speciation in natural environments, and an excellent system to explore the immediate consequences of allopolyploidy in a well-established historical and phylogenetic framework [9-11]. Hence, when comparing S. anglica to its parents, several studies [12-15] highlighted gene expression evolution, and epigenetic modifications such 
as DNA methylation alterations. Spartina species are regularly confronted with fluctuating environments: they are able to tolerate several hours of submersion in seawater, thanks to a developed aerenchyma [16] and efficient 'anaerobic respiration' capabilities [17]. They are also strongly tolerant to flooding [16,17], salt stress [18] and chemical pollution (e.g. PAHs, crude oil, heavy metals; see [19-24]).

Spartina species are recurrently exposed to oil spills [20,24]. In this context, we argued that allopolyploidy might contribute to Spartina tolerance to abiotic stress, such as tolerance to PAHs (Polycyclic Aromatic Hydrocarbons). PAHs are ubiquitous organic pollutants which represent a major concern in terms of ecotoxicology and public health [25-27]. PAH plants detoxification mechanisms are still poorly understood, but studies reported that plants are able to absorb, metabolize and/or degrade PAHs. Most organic xenobiotics are taken up by plant roots from soil, and translocated through the xylem to the shoot system for compartmentation and degradation $[28,29]$. Indeed, the uptake of PAHs by plant roots occurs through simple diffusion [29] or active transport [30].

In response to PAHs, plants are experiencing xenobiotics toxicity and associated oxidative stress damages. Xenobiotics affect the plant physiology by reducing major metabolic functions such as photosynthesis and respiration [31-33]. Moreover, PAHs are described to induce oxidative stress, through ROS (Reactive Oxygen Species) accumulation in plant cells, which in turn reduce plant development and growth, chlorophyll levels, and lead to trichome deformation and necrosis $[31,32,34]$. Oxidative stress damages induced by ROS under PAH stress are balanced by antioxidant enzymes, that are most likely limiting xenobiotic tolerance abilities [32]. More specifically, Dumas et al. [35] found that PAH accumulation in leaves inhibits electron transfer and photosynthesis within a few minutes, disrupting the energy transformation. Additionally, a microarray-based transcriptional profiling has established transcriptional reprogramming in Arabidopsis exposed to PAH $[35,36]$. These investigations under PAH-induced stress highlighted the importance of plant hormones such as ethylene [36] and other secondary metabolites [37] involved in defense pathways.

Introduced by Edwards et al. [38], the concept of 'xenome' was developed to characterize genes involved in tolerance mechanisms and metabolization of xenobiotics. In Arabidopsis, transcriptomic analysis under PAH-induced stress (plantlets exposed to phenanthrene) led to the identification of a gene set potentially involved in PAH sensing and detoxification [35]. PAH transformation involves oxidoreductases and hydrolases (including cytochromes P450: CYPs, peroxidases, dioxygenases and carboxylesterases) for xenobiotic solubilization. PAHs are then conjugated to endogenous molecules by the intervention of transferases, including glutathione-S- 
transferases (GSTs), glycosyltransferases (GTs) and malonyltransferases (MTs). These transformed molecules are then either neutralized by partnering with cell wall polymers such as lignin, stored in the vacuole through ATP-binding cassette (ABC) transporters, or exported out of the cell $[39,38]$. Plant response to xenobiotics occurs rapidly, and can be divided into three phases as (1) perception and signaling, (2) detoxification, and (3) degradation [40,35]. Most of our knowledge of the molecular and physiological mechanisms involved in xenobiotic tolerance relies on model systems such as Arabidopsis. Recently, Alvarez et al. [41] performed transcriptome profiling of resilient $S$. alterniflora natural populations exposed to crude oil, that provided some candidate genes involved in xenobiotic detoxification mechanisms.

In the present paper, we explored the Spartina model in order to examine the impact of interspecific hybridization and whole genome duplication (allopolyploidization) in the establishment of tolerance mechanisms to phenanthrene. Hence, we conducted comparative analyses of physiological changes in the parental species and the allopolyploid S. anglica under phenanthrene-induced stress, quantification and histochemical localization in leaf tissues. In parallel, we assessed changes of oxidative stress, and photosynthetic capacity (chlorophyll fluorescence) under phenanthrene-induced stress, and analyzed transcriptional changes of candidate genes involved in PAH detoxification pathways. The impact of allopolyploidy is subsequently discussed.

\section{MATERIALS AND METHODS}

\subsection{Plant materials}

We collected plants in various locations along the Brittany coastline (France). We sampled $S$. alterniflora in Hopital-Camfrout (Finistère, France), whereas S. maritima and S. anglica were collected at Le Hezo (Morbihan, France). We maintained plants transplanted in pots containing a mixture of soil and sand (70 \% and 30\%, respectively) in the greenhouse, under fluorescent light/dark regime of $16 / 8 \mathrm{~h}$, and an average ambient temperature of $20^{\circ} \mathrm{C}$.

\subsection{In vitro experimental design: culture conditions and phenanthrene treatments}

As germination of Spartina seeds is problematic (most particularly in S. maritima), we designed an in vitro protocol, using square leaf portions $(0.5-1 \mathrm{~cm}$ large $)$ placed in Petri dishes $(15 \times 15 \mathrm{~cm})$. We used phenanthrene (phe: $\mathrm{C}_{14} \mathrm{H}_{10}$ ), which is commonly reported as a PAH representative molecule. The molecular structure of phenanthrene is composed of three fused benzene rings, 
resulting in a low molecular weight, and efficient plant uptake. In crude oil, phenanthrene may represent up to $17 \%$ of total PAHs [42].

First, we incubated leaf tissues for $5 \mathrm{~min}$ in sodium hypochlorite (1.25\% active chlorine), and rinsed successively three times during $5 \mathrm{~min}$ in sterile water. We prepared Petri dishes with a solid growth medium (half-strength Murashige and Skoog: 0.5 MS; 0.8\% agar; $\mathrm{pH}=5.6$ ). These media were supplemented or not with phenanthrene from a stock solution dissolved in absolute ethanol at $200 \mathrm{mM}$, reaching concentrations of 0,100 and $400 \mu \mathrm{M}$ phe. Such treatments allowed us to compare stress tolerance levels between species (control and phenanthrene-induced stress conditions), or to assess more precise dose-response effects. We added identical volumes of ethanol alone to controls. This simplified procedure provides an optimal control of experimental conditions, phenanthrene being assimilated by foliar uptake, as explained by Smith and Jones [43]. We cultivated foliar tissues in Petri dishes in a phytotronic chamber with light/dark regime of $16 / 8 \mathrm{~h}$, temperature $22 / 18^{\circ} \mathrm{C}$. We conducted all experiments in three biological and technical replicates.

\subsection{Phenanthrene histolocalisation and quantification in foliar tissues}

\subsubsection{Phenanthrene histolocalisation}

We performed microscopic observations at the Cell Imaging platform (INRA Toulouse, France) on semi-thin sections of leaves treated with 0 and $400 \mu \mathrm{M}$ phe. Per treatment, we observed sections from nine leaf fragments by species (three biological replicates from distinct plants and technical triplicates). Phenanthrene fluorescence was detected using epifluorescence microscopy (Axiozoom V16, Zeiss). We also analyzed samples by confocal microscopy (LEICA SP2), by scanning along different wavelengths in the visible spectrum to identify specific emissions for phenanthrene and cellular components. Exited at $253 \mathrm{~nm}$, chlorophylls are observed in red, and phenanthrene in blue (emitting around 430 and $455 \mathrm{~nm}$ ). Phenanthrene fluorescence is distinguished from lignin autofluorescence which specifically emit at $472 \mathrm{~nm}$ (see Supplemental Fig. S2). Then, we used three-dimensional fluorescent reconstructions of leaf tissues to identify and specifically locate the xenobiotic into the cellular matrix.

\subsubsection{Phenanthrene quantification}

We estimated phenanthrene contents in Spartina tissues on leaf portions treated under $100 \mu \mathrm{M}$ phe. We first rinsed the harvested plant material (around $200 \mathrm{mg}$ of leaf tissues from at least three fragments from biological triplicates) in absolute ethanol and in distilled water. Samples were lyophilized for $72 \mathrm{~h}$, before being ground and weighted. We extracted organic matter samples 
using an accelerated solvent extractor (ASE 200, Dionex) with dichloromethane at $100^{\circ} \mathrm{C}$ under pressure. After evaporation, we diluted extracts with dichloromethane, and added quantification standards. Thereafter, we first performed fractionation by liquid chromatography, then we proceeded to identification and quantification of phenanthrene by gas chromatography and mass spectrometry (GC-MS).

\subsection{Physiological and biochemical analyses}

\subsubsection{Chlorophyll fluorescence}

We quantified chlorophyll fluorescence using a pulse amplitude-modulated fluorometer (JuniorPAM, Walz GmbH; Effeltrich, Germany). The device delivers a saturating pulse through a light guide; and collects different parameters related to the plant photochemistry. Photon absorption by chlorophyll molecules occurred at the excited state. By returning to their basal state, the resulting energy dissipated by the chlorophyll is either used for photosynthesis, dissipated as heat or reemitted as fluorescence [44]. These three processes are closely related to each other. Thus, determining a change in chlorophyll fluorescence is equivalent to identify a change in the yield of photochemical photosystem. Here we focus on photosystem II (PS II) and most particularly on two parameters as indices of photosynthetic capacity: $F_{O}$ (basic fluorescence yield) and $F m$ (maximal fluorescence yield). These indices represent minimum and maximum fluorescence values where reaction centers of PS II are respectively activated and inactivated, and $F v$ is defined as the difference between the two indices as the maximum variable fluorescence $(F v=F m-F o)$. $F v / F m$ normalized ratio is used to approximate the maximum photochemical quantum yield of PS II, to assess photosynthetic apparatus status in response to stress.

We performed measurements on leaf fractions (nine foliar fragments from biological and technical triplicates) cultivated 10 days under phenanthrene treatments $(0,100$ and $400 \mu \mathrm{M})$, and acclimated for $30 \mathrm{~min}$ in the dark before measurements, preventing PS II activity.

\subsubsection{Oxidative stress response: detection of superoxide radicals}

We used nitroblue tetrazolium (NBT; N6876, Sigma-Aldrich) to detect superoxide radicals $\mathrm{O}_{2}^{-}$ presence (ROS), according to the method described by Rao and Davis [45]. Leaf fragments grown 2 days on phenanthrene concentrations of 0 and $400 \mu \mathrm{M}$ (and even up to $800 \mu \mathrm{M}$ phe) were immersed and infiltrated under vacuum with $3.5 \mathrm{mg}^{-\mathrm{ml}^{-1}} \mathrm{NBT}$ staining solution in a potassium phosphate buffer $(10 \mathrm{mM})$. Per treatment, we observed at least nine foliar portions from biological and technical triplicates. Then, samples were bathed in an acetic acid-glycerol-ethanol (in respective volumes $1: 1: 3$ ) solution at $100^{\circ} \mathrm{C}$ for $5 \mathrm{~min}$, before being bleached in $80 \%$ acetone. 
NBT allowed the observation of $\mathrm{O}_{2}{ }^{-}$compounds which precipitate in blue color on plant tissues $[34,46]$. ROS production detected in leaf tissues reflects oxidative stress damages related to phenanthrene exposure. Leaves were then stored in a glycerol-ethanol solution (vol. 1:4) and photographed.

\subsection{In silico and gene expression analysis}

2.5.1. In silico search for candidate genes involved in phenanthrene stress response Recently, xenomic candidates involved in PAH tolerance mechanisms were identified in $A$. thaliana grown in controlled conditions [35], and compared with functionally annotated crude oil responsive genes in Spartina [41]. Here, we investigated homologous genes in Spartina using the reference transcriptomes assembled from leaves and roots of $S$. maritima and S. alterniflora $[47,48]$, in order to compare their expression profile in the parents and the allopolyploid under phenanthrene-induced stress in comparison to controlled conditions. Reference transcriptomes are composed of EST contigs resulting from assemblies of both short (Illumina) and long (Roche-454) reads from multiple cDNA libraries for each species (as described in $[47,48]$ ). Reads were assembled with $90 \%$ identity to build consensus sequences of homoeologs used as references that were functionally annotated. These transcriptomes resulted in 44,158 contigs in S. alterniflora and 60,644 contigs in $S$. maritima. To optimize the investigations, we also used genomic contigs assembled from short reads WGS (Whole Genome Shotgun) of S. maritima DNA libraries (A. Salmon \& M. L. Ainouche, unpublished).

Based on the model described by Edwards et al. [38], we identified 99 differentially expressed (DE) candidate xenome genes (Supplemental Fig. S3, [35]). Because xenobiotic signaling and sensing mechanisms are still poorly understood, we focused on genes specifically involved in xenobiotic detoxification, conjugation, putative transformation and metabolization processes. These genes are classified within GSTs, GTs, CYPs, MTs, and ABC transporters. They represent 80 out of the total 99 DE genes reported in Arabidopsis under phenanthrene exposure (see [35]). We chose to select among them a set of overexpressed genes that might have a significant impact on the physiological plant tolerance to xenobiotics. Thus, we recovered $52 \mathrm{~A}$. thaliana candidate genes (16 GSTs, 15 GTs, 15 CYPs, 1 MTs and 5 ABC transporters) for identifying their homologs in Spartina genomic and transcriptomic datasets.

In the present work, we aimed at identifying in Spartina the homologous xenome genes in Arabidopsis using full-length CDS sequences recovered from the TAIR database (TAIR10, http://www.arabidopsis.org). To improve our investigations, we also considered the rice genome (Oryza sativa 204, v7.0) available on Phytozome (http://phytozome.jgi.doe.gov). The pipeline 
depicting step by step the investigation process of candidate genes in Spartina is summarized in Supplemental Fig. S4. We first identified some candidates already functionally annotated in the Spartina transcriptomes [47,49]. O. sativa homologs to Arabidopsis candidates were also identified using rice genome annotations. In addition, we performed BLAST alignments (BLASTn and tBLASTx) of A. thaliana gene sequences and their $O$. sativa homologs on S. alterniflora and S. maritima transcriptomes, and $S$. maritima genomic contigs. Gene hits with an e.value lower than $10^{-6}$ and homologies higher than $80 \%$ over at least 70 bases (BLASTn) or 50 bases (tBLASTx) were retained for A. thaliana and Monocot species alignments, while homologies higher than $80 \%$ over at least 100 bases (BLASTn) or 80 bases (tBLASTx) were retained for $O$. sativa - Spartina alignments.

\subsubsection{GSTs phylogeny and gene expression analysis}

Glutathione-S-transferases (GSTs) are a family of enzymes involved in many detoxification processes, including xenobiotic metabolism and limiting oxidative stress [50-53]. In the present work, the expression of GSTs genes were used as an example to highlight how the xenome components are transcriptionally regulated in the context of allopolyploidy under phenanthreneinduced stress.

\section{- GSTs phylogeny and homology assessment:}

We used GST homologs identified in Spartina, Oryza, and Arabidopsis as queries through BLASTp [54] requests on sorghum (Sorghum bicolor 313, v3.1), maize (Zea mays 284, v5b) and Oropecium thomaeum (386, v1.0) full length protein databases (from Phytozome) to identify putative orthologous sequences. O. thomaeum like Spartina, is member of the Chloridoideae subfamily, and currently represents the most related sequenced genome published to date. We retained homologies presenting an e.value cutoff lower than $10^{-6}$, alignment lengths greater than 100 bases, and sequence identities of more than $60 \%$.

To confirm candidate GSTs identified in Spartina, we validated the presence of functional protein domains by HMMsearch (Hidden Markov Models, e.value threshold 10 ${ }^{-3}$ ) against Pfam-A (version 30.0, [55]). We excised GST domains recovered in Spartina contigs, and aligned sequences using the MAFFT software (version 7 with basic parameters; [56]) on GST CDS sequences from A. thaliana, O. sativa, S. bicolor, Z. mays and O. thomaeum. We subjected the resulting alignment to Gblocks [57] to parse poorly aligned positions. Then, we performed phylogenetic analysis using the IQ-TREE web server [58]. We used the Maximum Likelihood method for tree construction with the TVM+G substitution model, and performed ultrafast 
bootstrap analysis [59] on 10,000 replicates. We displayed phylogenetic tree using FigTree v1.4.3 (http://tree.bio.ed.ac.uk).

\section{- GSTs gene expression under phenanthrene-induced stress:}

We performed GST genes expression analysis on foliar fragments treated 10 days with phenanthrene concentrations of 0 and $400 \mu \mathrm{M}$. After sampling, we immediately froze leaves in liquid nitrogen. We proceeded to RNA extraction with Trizol reagent (Sigma), according to a protocol previously employed in Spartina [15,47]. This method consists in two successive cycles of precipitation, using isopropanol and absolute ethanol with sodium acetate $(3 \mathrm{M}, \mathrm{pH}=5.3)$. We quantified each RNA sample using Nanodrop Spectrophotometer ND 1000 (Nanodrop Technologies, ThermoFisher Scientific Inc.) and purified extracts with a Turbo DNA-free kit (Ambion, Life technologies) to remove DNA contaminations, before storage at $-80^{\circ} \mathrm{C}$. We used about 700 ng of total RNA per sample for first-strand cDNA synthesis (kit ThermoScript RTPCR, Invitrogen, Life technologies), and normalized cDNA concentrations to $5 \mathrm{ng} . \mu \mathrm{l}^{-1}$ before RTqPCR analysis.

Each amplified sample contained $0.5 \mathrm{ng}$ of cDNA, $5 \mu \mathrm{l}$ of PowerUp SYBR Green Master Mix (ThermoFisher), $1 \mu 1$ of specific Reverse and Forward primers $(5 \mu \mathrm{M})$, and sterile water for a final volume of $10 \mu \mathrm{l}$. We designed twenty base primers using Primer3 software (http://primer3.ut.ee/), with an expected amplicon size ranging between 100 and 200 bp, an average GC content of 55\% and a Tm close to $60^{\circ} \mathrm{C}$. We first tested primers on Spartina genomic DNA, and selected only single band amplifications for the quantification of gene expression.

In total, we analyzed the expression of 18 cDNA samples ( 3 species and 3 biological replicates from control and phe treated leaves) for each selected GST homologs ( 3 technical replicates were performed). We performed negative controls containing only sterile water, and 5 points dilution ranges with a pool of total cDNA (4 fold diluted steps from 0.5 to $2.10^{-3} \mathrm{ng}$. $\mu \mathrm{l}^{-1}$ ) for each target gene. We conducted quantitative PCR on the LifeCycler 480 II (Roche), using the following program: pre-incubation at $95^{\circ} \mathrm{C}$ for $10 \mathrm{~min}$, followed by 45 cycles of $10 \mathrm{sec}$ incubation at $95^{\circ} \mathrm{C}$, $10 \mathrm{sec}$ annealing at $58^{\circ} \mathrm{C}$, and $10 \mathrm{sec}$ elongation at $68^{\circ} \mathrm{C}$ followed by optical reading. We performed melting curves (fluorescence acquisition every $0.5^{\circ} \mathrm{C}$ from 65 to $95^{\circ} \mathrm{C}$ ) to check specificity of amplified fragments, and normalized levels of gene expression compared to the housekeeping gene glyceraldehyde-3-phosphate dehydrogenase (GA3PDH), which remains stable under these conditions [47,60]. This gene was amplified with the primer sequences AGAGTGCCTCGTCAAGGAGA (F) and CTCCCAAGCAATCCTCATGT (R). 


\subsection{Statistical analysis}

We performed statistics with the RStudio software [61]. We analyzed phenanthrene quantification in foliar tissues between species, using one-way analysis of variance (ANOVA; model parameters checked by Shapiro and Bartlett tests), and pairwise comparisons using the Duncan procedure (at 95\% confidence interval). We compared chlorophyll fluorescence data within species by KruskalWallis multiple comparison's rank test with Bonferroni correction. We tested gene expression levels between species and treatments with two-way ANOVAs (model parameters checked by Shapiro and Bartlett tests), and conducted pairwise comparisons using post hoc Tukey HSD. We performed alternative analysis when model parameters were invalid, using Kruskal and Wallis and pairwise Wilcoxon-Mann Whitney tests. We also conducted comparisons between allopolyploid's expression levels and MPV (Mid-Parent Value, expected under additive parental gene expression in the allopolyploid) between treatments using the same procedure.

\section{RESULTS}

\subsection{Phenanthrene absorption and histolocalisation}

We observed histological sections of semi-thin treated (400 $\mu \mathrm{M}$ phe) and untreated leaves cultivated in vitro for one week by epifluorescence microscopy (Fig. 1). We did not detect phenanthrene bright spots (blue fluorescent patches) in control leaves (Fig. 1A). In treated leaves, we observed phenanthrene by specific blue fluorescence in specific tissues depending on the species considered, as indicated by orange arrows in Fig. 1B. We confirmed that phenanthrene presence in cells of the leaf margins and the xylem in the three Spartina species, but we also found xenobiotic in sclerenchyma cells in S. alterniflora and S. anglica. It was present in the mesophilic achlorophyllous parenchyma in the allopolyploid, and in the sub-epidermal parenchyma in $S$. alterniflora. If distinguishing phenanthrene fluorescence from lignin autofluorescence is challenging, because they emit at partially overlapping wavelengths (between 450 and $500 \mathrm{~nm}$ for lignin and 420-480 $\mathrm{nm}$ for phenanthrene on samples exited at $253 \mathrm{~nm}$ ), phenanthrene specific emission spectra was detected at 420-440 nm which allowed to specifically identify the xenobiotic into the cellular matrix. In addition, more detailed analyses by confocal microscopy indicated that the presence of the pollutant is actually intracellular (see Fig. 1C), either cytosolic or vacuolar. These observations suggest that compartmentalization strategies could impact tolerance to xenobiotics of these three species. 
We used GC-MS for phenanthrene quantification in order to estimate the free phenanthrene content absorbed by leaf tissues (Fig. 2). After 10 days treatment (100 $\mu \mathrm{M}$ phe), we found $213.8 \pm$ $43.7 \mu \mathrm{g} . \mathrm{gDW}^{-1}$ phe in $S$. anglica, against $265.6 \pm 65.1$ in $S$. alterniflora and $468.8 \pm 23.0$ in $S$. maritima. These results revealed significantly higher phenanthrene content in S. maritima as compared to other species (p.value < 0.05). However, these data considered only phenanthrene in its toxic (and not chemically modified) form, which was not detected if subjected to any metabolic process (such as hydroxylation or glutathione conjugation).

\subsection{Photosynthetic activity under phenanthrene-induced stress}

After ten days of phenanthrene treatments $(0,100$ and $400 \mu \mathrm{M}$ phe), the average $F v / F m$ ratios estimated on control leaves $(0 \mu \mathrm{M}$ phe) were about $0.80 \pm 0.01$ for $S$. anglica and $0.72 \pm 0.03$ for S. alterniflora and S. maritima (Fig. 3). In S. anglica, we did not observe any significant treatment impact, even under high phenanthrene concentrations (whether on 100 or $400 \mu \mathrm{M}$ phe). However, the photosynthetic capacity of leaves was significantly reduced under $400 \mu \mathrm{M}$ phe in $S$. alterniflora $(F v / F m=0.47 \pm 0.06$, p.value $=0.0113)$ and from $100 \mu \mathrm{M}$ phe in $S$. maritima $(F v / F m$ $=0.52 \pm 0.08$, p.value $<0.05)$.

\subsection{Production of superoxide radicals under phenanthrene treatments}

Under control conditions, NBT detection shows slight blue coloration whatever the considered species (Fig. 4). These colorations are highly localized, suggesting that superoxide radical production (ROS) occurred on specific locations, probably because of leaf handling. In both $S$. alterniflora and S. anglica, phenanthrene treatment up to $800 \mu \mathrm{M}$ did not impact the production of superoxide radicals. In contrast, phenanthrene induced high oxidative stress on treated S. maritima leaves. This assumes that for such phenanthrene concentrations (whether 400 or $800 \mu \mathrm{M}$ phe), oxidative stress was limited in S. alterniflora and S. anglica whereas S. maritima seems more sensitive showing a pronounced reaction. Interestingly, we found that long time exposure to phenanthrene treatment (30 days under $100 \mu \mathrm{M}$ phe) did not induce any stress markers such as superoxide radical production and reduction of chlorophyll content in S. anglica. In contrast, the parental species S. alterniflora and S. maritima lost all chlorophyll contents and present severe senescent phenotype indicating cell death and tissue necrosis (results not shown).

\subsection{Candidate genes involved in phenanthrene tolerance}

In total, we validated 35 homologs in S. maritima and 31 in S. alterniflora using alignments to A. thaliana xenomic candidates. In complement, we added 11 homologies in $S$. maritima and 4 in $S$. 
alterniflora using alignments to $O$. sativa candidates. Among this set of putative homologs, we used 12 candidate genes only characterized within $S$. maritima genomic contigs for a final BLAST request (BLASTn and tBLASTx) on the transcriptome of $S$. alterniflora, which revealed four additional homologies on this species. Finally, we identified sequence homologies for 46 and 39 xenome candidate genes in S. maritima and S. alterniflora respectively (see Supplemental Table S1), related to $47 \mathrm{~A}$. thaliana locus (5 ABC transporters, 15 CYPs, 12 GSTs and 15 GTs). Among these genes, 38 present homologous sequences in both parental Spartina species.

In the present work, GSTs expression was used as an example to highlight how the xenome components are transcriptionally regulated in the context of the polyploidy under phenanthreneinduced stress. In total, we found 14 homologous sequences in the two parental Spartina species relative to $12 \mathrm{~A}$. thaliana-GSTs. Phylogenetic reconstruction by Maximum Likelihood based on $A$. thaliana, rice, maize, sorghum and $O$. thomaeum GST CDS sequence alignments is displayed on Fig. 5 (TVM substitution model; [58]). We used rice and Arabidopsis GSTs from the Omega family as outgroup to root the tree. A total of $10 \mathrm{CDS}$ sequences in A. thaliana, 14 in O. sativa, 19 in S. bicolor, 15 in Z. mays, 6 in O. thomaeum and 14 in Spartina were considered in the analysis. From this phylogenetic reconstruction, GST candidates retained in Spartina belong to the Omega GST gene family (Salt_2_contig34019, Salt_2_contig5351 and Smar_2_contig39931), and the plant specific Tau family. Spartina GST candidates were all similar to already known GSTs, and grouped within Tau or Omega GST families (ultrafast bootstrap value of 93). The tree topology distinguishes consistent specific clusters of Poaceae GSTs (highlighted in grey on the tree) as described by Jain et al. [62]. Among the 14 Spartina GST candidate sequences, we retained 6 contigs from $S$. maritima genomic sequencing after primer design (see Table 1, genes in bold on Supplemental Table S1) and followed their expressions patterns by RT-qPCR. However, we detected primer dimers on contig GA_02873 and removed the sequence from the analysis. Some of the technical replicates were invalidated and excluded from our analyses, but the limited extent of $\mathrm{Cp}$ (Crossing point) value distributions between them remain consistent. We checked PCR amplification efficiencies $\left(\mathrm{E}=10^{(-1 / \text { slope })}\right)$ by standard curves, and relative gene expression values were calculated with the $2^{-\Delta \mathrm{Cp}}$ method [63].

The relative expression ratios are presented in Fig. 6. Spartina GST homologous contigs identified present various expression patterns, depending on the species and the treatment applied. First, we compared expression profiles of GST contigs associated to S. alterniflora, S. maritima, and S. anglica within species between phenanthrene treatments $(0$ and $400 \mu \mathrm{M})$. In the parental species, the GST contigs selected did not display significant differential expressions considering the effect of ten days treatments on $400 \mu \mathrm{M}$ phe. In the neo-allopolyploid, similar patterns were observed for 
contigs GA_151485, GA_12349, GA_15763 and GA_263144. However, a significant downregulation is detected in response to phenanthrene in S. anglica for the GA_47211 contig, where relative ratios $\left( \pm \mathrm{SE}\right.$ ) decreased from $1.93 .10^{-2} \pm 3.53 \cdot 10^{-3}$ to $7.39 .10^{-3} \pm 2.53 \cdot 10^{-3}$ (Fig. 6A, p.value < 0.01). Across species, additive expression patterns were observed in S. anglica for contigs GA_151485, GA_12349 and GA_15763, as MPV relative ratios estimated under control ( $0 \mu \mathrm{M}$ phe) and treated conditions (400 $\mu \mathrm{M}$ phe) were respectively equivalent to those of the allopolyploid (p.value > 0.05). Conversely, we observed non-additive expressions in the allopolyploid for the contig GA_47211 with significant transgressive up-regulation (Fig. 6A, p.value < 0.001). We also observed that contig GA_263144 was expressed similarly to $S$. maritima in the allopolyploid under control condition (p.value $=0.50933$ ). However, under stress condition its expression appeared slightly induced as compared to the same parent (Fig. 6E, p.value $=0.02809$ ).

\section{DISCUSSION}

Understanding how plants adapt to challenging environment is a crucial question in evolutionary ecology. A growing body of evidence revealed the prominent role of whole genome duplication (polyploidy) in the plant diversification and adaptation [64]. In the present work, we explored the impact of allopolyploidization on plant xenobiotic tolerance in the saltmarsh lineage Spartina by comparing the recently formed allododecaploid species $S$. anglica to its parental species. This work represents the first comparative analyses performed to date between related Spartina species. The present analyses shed light on the detoxification processes involved in the three Spartina species exposed to phenanthrene in the context of natural allopolyploidization event, at the histochemical, biochemical and molecular levels.

\subsection{Tolerance to phenanthrene-induced stress in Spartina}

Phenanthrene histochemical investigations in Spartina revealed localized xenobiotic bright spots near the leaf margins, suggesting a potential establishment of evacuation processes by volatilization. Phenanthrene visualization in cells adjacent to the xylem tissues suggests that phenanthrene may be transported through the leaf by vascular elements as shown by Paterson et al. [65]. Furthermore, we confirmed by confocal microscopy that phenanthrene crossed the cell wall components and the plasmalemma, and was compartmented inside the cells (see Fig. 1C), either cytosolic or vacuolar. Intracellular presence of phenanthrene suggest that beyond being incorporated between cell walls, detoxification mechanisms of xenobiotics via metabolic pathways such as described in Edwards et al. [66] are ongoing in plant cells. However, differences observed 
between Spartina species are introducing distinct cellular specific compartmentalization strategies, which may affect detoxifying properties. In $S$. anglica and S. alterniflora, we also detected phenanthrene in sclerenchyma and parenchyma, in contrast with $S$. maritima where it is only reported near the cells of the xylem. Highly localized phenanthrene bright spots suggest that xenobiotics in S. alterniflora and S. anglica were transferred into supportive and storage tissues, maybe for degradation and accumulation.

Concerning ROS production analyses, the absence of superoxide radicals observed in S. anglica and $S$. alterniflora even under high phenanthrene contents ( $800 \mu \mathrm{M}$ phe) revealed high abilities of oxidative stress management. In contrast, Shiri et al. [34] recently analyzed the PAH sensitive species Arabidopsis thaliana and showed that ROS induction was detected at much lower phenanthrene concentrations $(25 \mu \mathrm{M}$ phe), supporting that phenanthrene stress levels used in our study highlights high tolerance abilities to PAHs in Spartina species.

\subsection{Identification of homologous xenome genes in Spartina}

Xenobiotic detoxification systems are still poorly understood in plants and have been explored in a few model systems $[67,66]$. Recent studies have explored the functional dynamics of the xenome in Arabidopsis under phenanthrene-induced stress [35,36,38], and comparisons with xenome responses to crude oil in natural S. alterniflora populations were performed [41]. Here, in silico detection of homologous xenome genes (as described in [35]) allowed an accurate identification of candidate genes involved in phenanthrene tolerance in Spartina. We retained a total of 38 genes from the A. thaliana xenome by homology searches in Spartina. Candidate xenome genes we identified represents putative phenanthrene detoxifying components in non-model Monocots such as Spartina which may be addressed in further analyses to study molecular mechanisms related to PAH detoxification.

We focused on the GST gene family which concentrate ubiquitous enzymes responsive to numerous stresses [51,52] to investigate the impact of allopolyploidization on xenome gene expression patterns under phenanthrene-induced stress. Several studies have demonstrated their role in the oxidative stress [68] by promoting antioxidant regeneration [69], or in xenobiotic detoxification by glutathione compounds conjugation [70]. Using available transcriptomic and genomic resources in the studied Spartina species [47,49] (A. Salmon \& M. L. Ainouche, unpublished), we identified 14 Spartina GST contigs. Three of them (Salt_2_contig34019, Salt_2_contig5351 and Smar_2_contig39931) are grouped among the Omega GST family. Others belong to the plant specific GST Tau family, a class already described to have a major role in several detoxification processes [71]. 
Expression patterns of five GSTs from the Tau family were analyzed (filled triangles in the tree Fig. 5). Among four selected GST candidate genes, expression profiles did not reveal significant changes under phenanthrene-induced stress in the three Spartina species. However, significant down-regulation in response to phenanthrene found in S. anglica (contig GA_47211; Fig. 6A) illustrates divergent regulation pathways opportunities under genome duplication event. While most of GST candidate genes analyzed did not present significant responses to phenanthrene, it is not clear how these genes may be involved in detoxification response to phenanthrene in Spartina, as suggested by Alvarez et al. [41]. However, Spartina and Arabidopsis belong to divergent lineages in Monocots and Eudicots respectively, which separated 170-220 MYA [72]. Thus, tolerance to organic xenobiotics may then involve distinct metabolic pathways and detoxification mechanisms, maybe through different transformation processes (Supplemental Fig. S3) recruiting other GSTs, GTs and malonyltransferases [36,66]. Interestingly, our phylogenetic analysis revealed Poaceae specific GST clades, as described by Jain et al. [62]. Hence, such genes may have acquired novel functions, as illustrated by the GST Spartina contigs which appeared quite distant from other GSTs in the phylogenetic analysis (see Fig. 5). Here, we only focused on GSTs as an example, but larger xenome profiling between species in controlled conditions are needed to clarify molecular mechanisms involved in phenanthrene detoxification in Spartina.

\subsection{Does allopolyploidy impact tolerance to xenobiotics?}

The recent formation of $S$. anglica during the end of the $19^{\text {th }}$ century offers a unique opportunity to explore the early evolutionary changes associated with the formation of a new allopolyploid species in natural populations, comparing the neo-allopolyploid to its parental species [12]. Morphological and ecological traits of the rapidly expanding neo-allopolyploid compared to its parental species have been thoroughly described [73,74].

Our comparative physiological analysis highlights that $S$. maritima is the most sensitive species to PAH-induced stress. The significant production of superoxide radicals in S. maritima leaves indicates that oxidative stress through superoxide production was pronounced in this species, contrasting with the tolerance exhibited by S. alterniflora and S. anglica, which developed stress tolerance abilities (Figure 2 and 4). Spartina maritima has reduced antioxidant scavenging capacities, thus increasing phenanthrene toxicity. Our findings also agree with the reduction in photosynthetic activity observed in S. maritima, as ROS are known to negatively impact photosynthetic rates [75]. Moreover, phenanthrene uptake assays performed in leaf tissues revealed contrasted free phenanthrene contents among species, the lowest amount being quantified in the neo-allopolyploid $S$. anglica and the highest in $S$. maritima. In complement, additional 
histolocalisation of xenobiotics into S. alterniflora and S. anglica plant tissues (sclerenchyma and parenchyma) may be related with enhanced detoxification mechanisms.

Hence, we provide experimental data supporting that enhanced xenobiotic tolerance are found in $S$. anglica and $S$. alterniflora in contrast with pronounced sensitivity in S. maritima, consistently with $F v / F m$ ratio measured under $100 \mu \mathrm{M}$ phe, significantly reduced in the paternal parent in contrast to other species. Altogether, these findings may be related to ecological traits of the compared species. S. anglica and S. alterniflora are described as vigorous invasive species [7681] whereas $S$. maritima is restricted to its native area along European and African coast where its seems declining in some sites (e.g. England; [82]). The three species usually colonize low mash habitats and mudflats with lower salinity ranges [83-85] but S. anglica and S. alterniflora are also present in areas more frequently flooded or high marsh zones, and may be exposed to stronger salt and drought levels [77,86-88].

The photosynthetic system is a key indicator to estimate plant tolerance to biotic and abiotic stress. Previous studies showed that $S$. alterniflora photosynthetic rates were severely affected when growing in PAH contaminated soils [89,90]. Our study reveals that phenanthrene stress tolerance increased with ploidy level in Spartina, since S. anglica maintained high chlorophyll fluorescence levels under phenanthrene-induced stress in contrast to its parental species. Under phenanthrene treatment, we found that photosynthetic activity was significantly reduced in the parental species. Thus, it seems that such phenanthrene concentrations (400 $\mu \mathrm{M}$ phe) did not affect $S$. anglica photosynthetic apparatus, as opposed to the parental species. Moreover, by comparing S. anglica to $S$. alterniflora we observed different patterns of phenanthrene localization, mainly accumulated in the sub-epidermal parenchyma in S. alterniflora, in contrast to the mesophilic parenchyma in $S$. anglica.

Differences in tolerance levels between species were detected through enhanced tolerance reported in S. anglica, compared to its parental species (Figure 1 and 3), consistently with long term tolerance experiments (30 days under $100 \mu \mathrm{M}$ phe) which highlight senescent phenotypes in the parental species while $S$. anglica did not exhibit any phenotypic stress markers. This suggests that the allopolyploid S. anglica cope with chronic organic xenobiotic exposure through efficient metabolic detoxification pathways. As the recent origin and the low inter-individual genetic diversity related to clonal propagation reported in S. anglica are limiting the effects of selection acting on different genotypes [9-11], it is reasonable to speculate that tolerance to PAHs results from immediate evolutionary effects of WGD. To our knowledge, the present work demonstrates 
for the first time that allopolyploidy may enhance tolerance to organic pollutants. Nevertheless, one can keep in mind that other mechanisms resulting from the species history might interact.

Allopolyploidy highly impacts gene expression (i.e. deviation from expected parental additivity), and may have critical adaptive impact on newly formed polyploid species [91]. Previous transcriptomic investigations in polyploid Spartina were performed in both controlled [15] and natural [92] conditions and revealed various expression patterns in $S$. anglica compared to its parental species. In the present study, expression of the GST genes involved in A. thaliana xenobiotic tolerance was explored. Transgressive up-regulation was recorded for contig GA_47211 with respect to MPV and parental expression patterns. Non-additive expression was also exhibited by the contig GA_263144 in control condition, while expression in S. anglica was statistically equivalent to its expression in S. maritima, but differed from the MPV and from its expression in S. alterniflora (expression dominance mimicking the paternal expression pattern). Interestingly, paternal dominance appeared to be lost under stress conditions, as expression in $S$. anglica was higher and additive with respect to parental expression patterns (statistically equivalent to the MPV).

Allopolyploidy is a combination of two different evolutionary events, i.e. the merger of divergent genomes (resulting from hybridization) and whole genome duplication (resulting from polyploidy) that shape the newly formed allopolyploid genome $[13,15]$. Thus, changes detected in $S$. anglica may reflect one or both evolutionary events. Additional comparative analyses of the xenome in the F1 hybrid (S. $x$ townsendii) will help deciphering the relative contributions of hybridization and/or genome doubling per se. Expression plasticity exploiting the union of divergent expression and regulatory networks inherited from S. maritima and S. alterniflora which diverged since 2-4 MYA [93], combined with important epigenetic alterations [13,14], most likely represent a key component in S. anglica enhanced tolerance to xenobiotics. As halophytes represent an emerging trend in phytoremediation [94], our results are supporting using S. anglica for such purpose on organic pollutants.

\section{Author contributions}

A.E, A.S and M.A. designed the experiments. A.E., O.L. and A.C.R. performed the experiments. A.E., A.S., M.A. and A.C.R. analyzed data. A.E., A.S., M.A. and A.C.R. wrote the article.

\section{Acknowledgements}

This work was supported by the Ministère de l'Enseignement Supérieur et de la Recherche, by the CNRS, and the Observatoire des Sciences et de l'Univers de Rennes (OSUR). The authors would 
like to knowledge Olivier Catrice with his assistance for the achievement of confocal microscopy analysis. We also thank two anonymous reviewers for their constructive comments.

Conflicts of interest: 'none' 


\section{REFERENCES}

[1] D.E. Soltis, V.A. Albert, J. Leebens-Mack, C.D. Bell, A.H. Paterson, C. Zheng, D. Sankoff, C.W. de Pamphilis, P.K. Wall, P.S. Soltis, Polyploidy and angiosperm diversification, Am. J. Bot. 96 (2009) 336-348.

[2] L. Comai, The advantages and disadvantages of being polyploid, Nat. Rev. Genet. 6 (2005) 836-846.

[3] A. Madlung, J.F. Wendel, Genetic and epigenetic aspects of polyploid evolution in plants, Cytogenet. Genome Res. 140 (2013) 270-285.

[4] P.S. Soltis, D.B. Marchant, Y. Van de Peer, D.E. Soltis, Polyploidy and genome evolution in plants, Curr. Opin. Genet. Dev. 35 (2015) 119-125.

[5] K. Alix, P.R. Gérard, T. Schwarzacher, J.S. (Pat) Heslop-Harrison, Polyploidy and interspecific hybridization: partners for adaptation, speciation and evolution in plants, Ann. Bot. 120 (2017) 183-194.

[6] J.J. Doyle, L.E. Flagel, A.H. Paterson, R.A. Rapp, D.E. Soltis, P.S. Soltis, J.F. Wendel, Evolutionary genetics of genome merger and doubling in plants, Annu. Rev. Genet. 42 (2008) 443-461.

[7] P.M. Peterson, K. Romaschenko, Y.H. Arrieta, J.M. Saarela, A molecular phylogeny and new subgeneric classification of Sporobolus; (Poaceae: Chloridoideae: Sporobolinae), Taxon. 63 (2014) 1212-1243.

[8] S. Lowe, M. Browne, S. Boudjelas, M. De Poorter, 100 of the world's worst invasive alien species: A selection from the Global invasive species database., Invasive Species Spec. Group ISSG Spec. Group Species Surviv. Comm. SSC World Conserv. Union IUCN. First published as special lift-out in Aliens 12 (2000) 12pp.

[9] M.L. Ainouche, A. Baumel, A. Salmon, G. Yannic, Hybridization, polyploidy and speciation in Spartina (Poaceae), New Phytol. 161 (2003) 165-172.

[10] M. Ainouche, H. Chelaifa, J. Ferreira, S. Bellot, A. Ainouche, A. Salmon, Polyploid evolution in Spartina: Dealing with highly redundant hybrid genomes, in: P.S. Soltis, D.E. Soltis (Eds.), Polyploidy Genome Evol., Springer Berlin Heidelberg, Berlin, Heidelberg, 2012: pp. 225243.

[11] A. Baumel, M.L. Ainouche, J.E. Levasseur, Molecular investigations in populations of Spartina anglica C.E. Hubbard (Poaceae) invading coastal Brittany (France), Mol. Ecol. 10 (2001) 1689-1701.

[12] M.L. Ainouche, A. Baumel, A. Salmon, Spartina anglica C. E. Hubbard: a natural model system for analysing early evolutionary changes that affect allopolyploid genomes: evolution of the Spartina anglica, Biol. J. Linn. Soc. 82 (2004) 475-484. 
[13] A. Salmon, M.L. Ainouche, J.F. Wendel, Genetic and epigenetic consequences of recent hybridization and polyploidy in Spartina (Poaceae), Mol. Ecol. 14 (2005) 1163-1175.

[14] C. Parisod, A. Salmon, T. Zerjal, M. Tenaillon, M.-A. Grandbastien, M. Ainouche, Rapid structural and epigenetic reorganization near transposable elements in hybrid and allopolyploid genomes in Spartina, New Phytol. 184 (2009) 1003-1015.

[15] H. Chelaifa, A. Monnier, M. Ainouche, Transcriptomic changes following recent natural hybridization and allopolyploidy in the salt marsh species Spartina $\times$ townsendii and Spartina anglica (Poaceae), New Phytol. 186 (2010) 161-174.

[16] B.R. Maricle, R.W. Lee, Aerenchyma development and oxygen transport in the estuarine cordgrasses Spartina alterniflora and S. anglica, Aquat. Bot. 74 (2002) 109-120.

[17] B.R. Maricle, J.J. Crosier, B.C. Bussiere, R.W. Lee, Respiratory enzyme activities correlate with anoxia tolerance in salt marsh grasses, J. Exp. Mar. Biol. Ecol. 337 (2006) 30-37.

[18] J.B. Adams, G.C. Bate, Ecological implications of tolerance of salinity and inundation by Spartina maritima, Aquat. Bot. 52 (1995) 183-191.

[19] A.W. Watts, T.P. Ballestero, K.H. Gardner, Uptake of polycyclic aromatic hydrocarbons (PAHs) in salt marsh plants Spartina alterniflora grown in contaminated sediments, Chemosphere. 62 (2006) 1253-1260.

[20] I.A. Mendelssohn, G.L. Andersen, L.P. Rozas, Oil impacts on coastal wetlands: Implications for the Mississippi river delta ecosystem after the deepwater horizon oil spill, BioScience. 62 (2012) 562-574.

[21] S. Redondo-Gomez, Bioaccumulation of heavy metals in Spartina, Funct. Plant Biol. (2013).

[22] G. Curado, A.E. Rubio-Casal, E. Figueroa, J.M. Castillo, Potential of Spartina maritima in restored salt marshes for phytoremediation of metals in a highly polluted estuary, Int. J. Phytoremediation. 16 (2014) 1209-1220.

[23] P. Biber, W. Wu, M. Peterson, Z. Liu, L. Pham, Oil contamination in Mississippi salt marsh habitats and the impacts to Spartina alterniflora photosynthesis, in: J. Alford, M. Peterson, C. Green (Eds.), Impacts Oil Spill Disasters Mar. Habitats Fish. N. Am., CRC Press, 2014: pp. $133-172$.

[24] A. Bergen, C. Alderson, R. Bergfors, C. Aquila, M.A. Matsil, Restoration of a Spartina alterniflorasalt marsh following a fuel oil spill, New York City, NY, Wetl. Ecol. Manag. 8 (2000) 185-195. doi:10.1023/A:1008496519697.

[25] C.H. Chaîneau, J.L. Morel, J. Oudot, Phytotoxicity and plant uptake of fuel oil hydrocarbons, J. Environ. Qual. 26 (1997) 1478. 
[26] P.J. Harvey, B.F. Campanella, P.M.L. Castro, H. Harms, E. Lichtfouse, A.R. Schäffner, S. Smrcek, D. Werck-Reichhart, Phytoremediation of polyaromatic hydrocarbons, anilines and phenols, Environ. Sci. Pollut. Res. 9 (2002) 29-47.

[27] B.M. Greenberg, PAH Interactions with plants: Uptake, toxicity and phytoremediation, in: P.E.T. Douben (Ed.), PAHs Ecotoxicological Perspect., John Wiley \& Sons, Ltd, 2003: pp. 263273.

[28] Y. Gao, L. Zhu, Plant uptake, accumulation and translocation of phenanthrene and pyrene in soils, Chemosphere. 55 (2004) 1169-1178.

[29] E. Pilon-Smits, Phytoremediation, Annu. Rev. Plant Biol. 56 (2005) 15-39.

[30] X. Zhan, X. Zhang, X. Yin, H. Ma, J. Liang, L. Zhou, T. Jiang, G. Xu, H+/phenanthrene symporter and aquaglyceroporin are implicated in phenanthrene uptake by wheat (L.) roots, J. Environ. Qual. 41 (2012) 188.

[31] M. Alkio, T.M. Tabuchi, X. Wang, A. Colon-Carmona, Stress responses to polycyclic aromatic hydrocarbons in Arabidopsis include growth inhibition and hypersensitive response-like symptoms, J. Exp. Bot. 56 (2005) 2983-2994.

[32] H. Liu, D. Weisman, Y. Ye, B. Cui, Y. Huang, A. Colón-Carmona, Z. Wang, An oxidative stress response to polycyclic aromatic hydrocarbon exposure is rapid and complex in Arabidopsis thaliana, Plant Sci. 176 (2009) 375-382.

[33] C. Sulmon, G. Gouesbet, F. Ramel, F. Cabello-Hurtado, C. Penno, N. Bechtold, I. Couée, A.E. Amrani, Carbon dynamics, development and stress responses in Arabidopsis: Involvement of the APL4 subunit of ADP-Glucose pyrophosphorylase (starch synthesis), PLoS ONE. 6 (2011).

[34] M. Shiri, M. Rabhi, C. Abdelly, A.E. Amrani, The halophytic model plant Thellungiella salsuginea exhibited increased tolerance to phenanthrene-induced stress in comparison with the glycophitic one Arabidopsis thaliana: Application for phytoremediation, Ecol. Eng. 74 (2015) 125-134.

[35] A.-S. Dumas, L. Taconnat, E. Barbas, G. Rigaill, O. Catrice, D. Bernard, A. Benamar, D. Macherel, A. El Amrani, R. Berthomé, Unraveling the early molecular and physiological mechanisms involved in response to phenanthrene exposure, BMC Genomics. 17 (2016).

[36] D. Weisman, M. Alkio, A. Colón-Carmona, Transcriptional responses to polycyclic aromatic hydrocarbon-induced stress in Arabidopsis thaliana reveal the involvement of hormone and defense signaling pathways, BMC Plant Biol. 10 (2010) 59.

[37] A.C. Singer, D.E. Crowley, I.P. Thompson, Secondary plant metabolites in phytoremediation and biotransformation, Trends Biotechnol. 21 (2003) 123-130.

[38] R. Edwards, D.P. Dixon, I. Cummins, M. Brazier-Hicks, M. Skipsey, New perspectives on the metabolism and detoxification of synthetic compounds in plants, in: P. Schröder, C.D. Collins (Eds.), Org. Xenobiotics Plants, Springer Netherlands, Dordrecht, 2011: pp. 125-148. 
[39] G. Taguchi, T. Ubukata, H. Nozue, Y. Kobayashi, M. Takahi, H. Yamamoto, N. Hayashida, Malonylation is a key reaction in the metabolism of xenobiotic phenolic glucosides in Arabidopsis and tobacco: Phenolic-xenobiotics metabolism in Arabidopsis, Plant J. 63 (2010) 1031-1041.

[40] A. El Amrani, A.-S. Dumas, L.Y. Wick, E. Yergeau, R. Berthomé, “Omics” Insights into PAH Degradation Toward Improved Green Remediation Biotechnologies, Environ. Sci. Technol. 49 (2015) 11281-11291.

[41] M. Alvarez, J. Ferreira de Carvalho, A. Salmon, M.L. Ainouche, A. Cavé-Radet, A. El Amrani, T.E. Foster, S. Moyer, C.L. Richards, Transcriptome response to the Deepwater Horizon oil spill identifies novel candidate genes for oil tolerance in natural populations of the foundation plant Spartina alterniflora, Mol. Ecol. (2018).

[42] Z. Liu, J. Liu, Q. Zhu, W. Wu, The weathering of oil after the Deepwater Horizon oil spill: insights from the chemical composition of the oil from the sea surface, salt marshes and sediments, Environ. Res. Lett. 7 (2012) 035302.

[43] K.E.C. Smith, K.C. Jones, Particles and vegetation: implications for the transfer of particle-bound organic contaminants to vegetation, Sci. Total Environ. 246 (2000) 207-236.

[44] A. Narayan, M. Misra, R. Singh, Chlorophyll fluorescence in plant biology, in: P.D.A.N. Misra (Ed.), Biophysics, InTech, 2012.

[45] M.V. Rao, K.R. Davis, Ozone-induced cell death occurs via two distinct mechanisms in Arabidopsis: the role of salicylic acid, Plant J. 17 (1999) 603-614.

[46] F. Ramel, C. Sulmon, F. Cabello-Hurtado, L. Taconnat, M.-L. Martin-Magniette, J.-P. Renou, A. El Amrani, I. Couée, G. Gouesbet, Genome-wide interacting effects of sucrose and herbicide-mediated stress in Arabidopsis thaliana: novel insights into atrazine toxicity and sucrose-induced tolerance, BMC Genomics. 8 (2007) 450.

[47] J. Ferreira de Carvalho, J. Poulain, C. Da Silva, P. Wincker, S. Michon-Coudouel, A. Dheilly, D. Naquin, J. Boutte, A. Salmon, M. Ainouche, Transcriptome de novo assembly from next-generation sequencing and comparative analyses in the hexaploid salt marsh species Spartina maritima and Spartina alterniflora (Poaceae), Heredity. 110 (2013) 181-193.

[48] J. Boutte, B. Aliaga, O. Lima, J. Ferreira de Carvalho, A. Ainouche, J. Macas, M. Rousseau-Gueutin, O. Coriton, M. Ainouche, A. Salmon, Haplotype detection from Next Generation Sequencing in high ploidy-level species: 45S rDNA gene copies in the hexaploid Spartina maritima, G3 GenesGenomesGenetics. (2015).

[49] J. Boutte, C.J. Ferreira de, M. Rousseau-Gueutin, J. Poulain, C. Da Silva, P. Wincker, M. Ainouche, A. Salmon, Reference transcriptomes and detection of duplicated copies in hexaploid and allododecaploid Spartina species (Poaceae), Genome Biol. Evol. (2016) evw209. 
[50] A.S. Milligan, A. Daly, M.A.J. Parry, P. Lazzeri, I. Jepson, The expression of a maize glutathione S-transferase gene in transgenic wheat confers herbicide tolerance, both in planta and in vitro, Mol. Breed. 7 (2001) 301-315.

[51] N.E. Labrou, A.C. Papageorgiou, O. Pavli, E. Flemetakis, Plant GSTome: structure and functional role in xenome network and plant stress response, Curr. Opin. Biotechnol. 32 (2015) 186-194.

[52] K. Nahar, M. Hasanuzzaman, M. Fujita, Physiological roles of glutathione in conferring abiotic stress tolerance to plants, in: N. Tuteja, S.S. Gill (Eds.), Abiotic Stress Response Plants, Wiley-VCH Verlag GmbH \& Co. KGaA, Weinheim, Germany, 2016: pp. 155-184.

[53] B.P. DeRidder, Induction of Glutathione S-Transferases in Arabidopsis by herbicide safeners, PLANT Physiol. 130 (2002) 1497-1505.

[54] S.F. Altschul, W. Gish, W. Miller, E.W. Myers, D.J. Lipman, Basic local alignment search tool, J. Mol. Biol. 215 (1990) 403-410.

[55] R.D. Finn, P. Coggill, R.Y. Eberhardt, S.R. Eddy, J. Mistry, A.L. Mitchell, S.C. Potter, M. Punta, M. Qureshi, A. Sangrador-Vegas, G.A. Salazar, J. Tate, A. Bateman, The Pfam protein families database: towards a more sustainable future, Nucleic Acids Res. 44 (2016) D279-D285.

[56] K. Katoh, D.M. Standley, MAFFT Multiple sequence alignment software version 7: Improvements in performance and usability, Mol. Biol. Evol. 30 (2013) 772-780.

[57] G. Talavera, J. Castresana, K. Kjer, R. Page, J. Sullivan, Improvement of phylogenies after removing divergent and ambiguously aligned blocks from protein sequence alignments, Syst. Biol. 56 (2007) 564-577.

[58] L.-T. Nguyen, H.A. Schmidt, A. von Haeseler, B.Q. Minh, IQ-TREE: A Fast and Effective Stochastic Algorithm for Estimating Maximum-Likelihood Phylogenies, Mol. Biol. Evol. 32 (2015) 268-274.

[59] B.Q. Minh, M.A.T. Nguyen, A. von Haeseler, Ultrafast Approximation for Phylogenetic Bootstrap, Mol. Biol. Evol. 30 (2013) 1188-1195.

[60] N. Baisakh, P.K. Subudhi, N.P. Parami, cDNA-AFLP analysis reveals differential gene expression in response to salt stress in a halophyte Spartina alterniflora Loisel, Plant Sci. 170 (2006) 1141-1149.

[61] R Core Team, R: a language and environement for statistical computing, $\mathrm{R}$ foundation for statistical computing, Vienne (Autriche), 2015. http://www.R-project.org/.

[62] M. Jain, C. Ghanashyam, A. Bhattacharjee, Comprehensive expression analysis suggests overlapping and specific roles of rice glutathione S-transferase genes during development and stress responses, BMC Genomics. 11 (2010) 73.

[63] M.W. Pfaffl, A new mathematical model for relative quantification in real-time RT-PCR, Nucleic Acids Res. 29 (2001) 45. 
[64] Y. Van de Peer, E. Mizrachi, K. Marchal, The evolutionary significance of polyploidy, Nat. Rev. Genet. 18 (2017) 411-424.

[65] S. Paterson, D. Mackay, C. McFarlane, A model of organic chemical uptake by plants from soil and the atmosphere, Environ. Sci. Technol. 28 (1994) 2259-2266.

[66] R. Edwards, M. Brazier-Hicks, D.P. Dixon, I. Cummins, Chemical manipulation of antioxidant defences in plants, in: Adv. Bot. Res., Elsevier, 2005: pp. 1-32.

[67] H. Sandermann, Plant metabolism of xenobiotics, Trends Biochem. Sci. 17 (1992) 82-84.

[68] P.G. Sappl, A.J. Carroll, R. Clifton, R. Lister, J. Whelan, A. Harvey Millar, K.B. Singh, The Arabidopsis glutathione transferase gene family displays complex stress regulation and cosilencing multiple genes results in altered metabolic sensitivity to oxidative stress: Genomic and reverse genetic analysis of plant GSTs, Plant J. 58 (2009) 53-68.

[69] P.-A. Lallement, B. Brouwer, O. Keech, A. Hecker, N. Rouhier, The still mysterious roles of cysteine-containing glutathione transferases in plants, Front. Pharmacol. 5 (2014).

[70] D.P. Dixon, R. Edwards, Glutathione Transferases, Arab. Book. 8 (2010).

[71] D.P. Dixon, B.G. Davis, R. Edwards, Functional Divergence in the Glutathione transferase superfamily in plants. Identification of two classes with putative functions in redox homeostasis in Arabidopsis thaliana, J. Biol. Chem. 277 (2002) 30859-30869.

[72] Y.-W. Yang, K.-N. Lai, P.-Y. Tai, W.-H. Li, Rates of nucleotide substitution in angiosperm mitochondrial DNA sequences and dates of divergence between Brassica and other angiosperm lineages, J. Mol. Evol. 48 (1999) 597-604.

[73] C.J. Marchant, Evolution in Spartina (Gramineae): I. The history and morphology of the genus in Britain, J. Linn. Soc. Lond. Bot. 60 (1967) 1-24.

[74] A.J. Gray, D.F. Marshall, A.F. Raybould, A Century of Evolution in Spartina anglica, in: Adv. Ecol. Res., Elsevier, 1991: pp. 1-62.

[75] A.R. Reddy, A.S. Raghavendra, Photooxidative stress, in: K.. Madhava Rao, A.S. Raghavendra, K. Janardhan Reddy (Eds.), Physiol. Mol. Biol. Stress Toler. Plants, Kluwer Academic Publishers, Dordrecht, 2006: pp. 157-186.

[76] Q. Wang, S.-Q. An, Z.-J. Ma, J.-K. Chen, B. Li, Invasive Spartina alterniflora: biology, ecology and management, Acta Phytotaxon. Sin. 44 (2006) 559.

[77] C.M. Anderson, M. Treshow, A Review of Environmental and Genetic Factors That Affect Height in Spartina alterniflora Loisel. (Salt Marsh Cord Grass), Estuaries. 3 (1980) 168.

[78] J.D. Thompson, The biology of an invasive plant, 41 (1991) 393-401.

[79] J. Adams, E. van Wyk, T. Riddin, First record of Spartina alterniflora in southern Africa indicates adaptive potential of this saline grass, Biol. Invasions. 18 (2016) 2153-2158. 
[80] M. Ainouche, A. Gray, Invasive Spartina: lessons and challenges, Biol. Invasions. 18 (2016) 2119-2122.

[81] W.H. Elmer, R.E. Marra, H. Li, B. Li, Incidence of Fusarium spp. on the invasive Spartina alterniflora on Chongming Island, Shanghai, China, Biol. Invasions. 18 (2016) 2221-2227.

[82] A.F. Raybould, A.J. Gray, M.J. Lawrence, D.F. Marshall, The evolution of Spartina anglica C. E. Hubbard (Gramineae): genetic variation and status of the parental species in Britain, Biol. J. Linn. Soc. 44 (1991) 369-380.

[83] M.D. Bertness, Zonation of Spartina patens and Spartina alterniflora in New England salt marsh, Ecology. 72 (1991) 138.

[84] J.M. Castillo, L. Fernandez-Baco, E.M. Castellanos, C.J. Luque, M.E. FigUeroa, A.J. Davy, Lower limits of Spartina densiflora and S. maritima in a Mediterranean salt marsh determined by different ecophysiological tolerances, J. Ecol. 88 (2000) 801-812.

[85] S.D. Hacker, D. Heimer, M.N. Dethier, A marine plant (Spartina anglica) invades widely varying habitats: Potential mechanisms of invasion and control, Biol. Invasions. 3 (2001) 211217.

[86] C.L. Richards, S.C. Pennings, L.A. Donovan, Habitat range and phenotypic variation in salt marsh plants, Plant Ecol. 176 (2005) 263-273.

[87] C.L. Richards, J.L. Hamrick, L.A. Donovan, R. Mauricio, Unexpectedly high clonal diversity of two salt marsh perennials across a severe environmental gradient, Ecol. Lett. 7 (2004) 1155-1162.

[88] J. Rozema, H. Gude, G. Pollak, An ecophysiological study of the salt secretion of four halophytes, New Phytol. 89 (1981) 201-217.

[89] S.R. Pezeshki, R.D. De Laune, Effect of crude oil on gas exchange functions of Juncus roemerianus and Spartina alterniflora, Water. Air. Soil Pollut. 68 (1993) 461-468.

[90] Q. Lin, I.A. Mendelssohn, Evaluation of tolerance limits for restauration anf phytoremediation with Spartina alterniflora in crude oil contaminated coastal salt marshes, Int. Oil Spill Conf. Proc. 2008 (2008) 869-873.

[91] M.L. Ainouche, J.F. Wendel, Polyploid speciation and genome evolution: Lessons from recent allopolyploids, in: P. Pontarotti (Ed.), Evol. Biol. Genome Evol. Speciat. Coevol. Orig. Life, Springer International Publishing, Cham, 2014: pp. 87-113.

[92] J. Ferreira de Carvalho, J. Boutte, P. Bourdaud, H. Chelaifa, K. Ainouche, A. Salmon, M. Ainouche, Gene expression variation in natural populations of hexaploid and allododecaploid Spartina species (Poaceae), Plant Syst. Evol. (2017).

[93] M. Rousseau-Gueutin, S. Bellot, G.E. Martin, J. Boutte, H. Chelaifa, O. Lima, S. MichonCoudouel, D. Naquin, A. Salmon, K. Ainouche, M. Ainouche, The chloroplast genome of the 
hexaploid Spartina maritima (Poaceae, Chloridoideae): Comparative analyses and molecular dating, Mol. Phylogenet. Evol. 93 (2015) 5-16.

[94] E. Manousaki, N. Kalogerakis, Halophytes-An Emerging Trend in Phytoremediation, Int. J. Phytoremediation. 13 (2011) 959-969.

[95] W.S. Alves, E.A. Manoel, N.S. Santos, R.O. Nunes, G.C. Domiciano, M.R. Soares, Detection of polycyclic aromatic hydrocarbons (PAHs) in Medicago sativa L. by fluorescence microscopy, Micron. 95 (2017) 23-30.

\section{FIGURES AND TABLES}

Figure 1. Histological observation of cross sections of Spartina leaves by confocal and epifluorescence microscopy. A: Phe accumulated in the cells was detected by specific blue fluorescent bright spots. In control leaves, no phe specific fluorescence was observed (scale bars $=50 \mu \mathrm{m}$ ). B: Phe locations are different between species treated with $400 \mu \mathrm{M}$ phe (scale bars $=100 \mu \mathrm{m})$. In the three species, phe was detected in the neighboring cells of the xylem. Phe was also specifically found in the sclerenchyma cells close to leaf margins, and in the mesophilic achlorophyllous parenchyma in S. anglica. In S. alterniflora, it is also present in the parenchyma cells and the sub-epidermal sclerenchyma. C: Phenanthrene colocalization into Spartina leaf plant tissues by confocal microscopy (scale bar $=25 \mu \mathrm{m}$.). Detection of the phenanthrene specific emission spectrum allowed us to identify the pollutant by assigning it a violet coloration. Confocal microscopy pics clearly distinguish phenanthrene (1) intracellular presence inside the cell walls (2), and chlorophyll fluorescence in red (3).

Figure 2. Phe concentrations in Spartina leaves cultivated 10 days on medium containing 100 $\mu \mathrm{M}$ phe. Means are calculated from three biological replicates; bars correspond to standard errors. The different letters are corresponding to significant concentration differences according to Duncan's test (p.value $<0.05$ ).

Figure 3. $F v / F m$ ratio under phenanthrene treatment $(0,100$ and $400 \mu \mathrm{M})$, an index of the maximum quantum yield of PS II for each of the three species of Spartina. Measurements were carried out after 10 days of phe treatment. Means are calculated from three biological replicates; bars correspond to the standard errors. Values annotated with different letters by species are significantly different according to Kruskal-Wallis multiple comparison's test (with Bonferroni correction, p.value $<0.05$ ). 
Figure 4. Visualization of superoxide radical production upon leaves NBT infiltrations. Spartina leaves were cultivated 2 days in medium containing 0, 400 and up to $800 \mu \mathrm{M}$ phe. Superoxide radicals were detected by the presence of blue spots. Light blue spots were visible on S. anglica and S. alterniflora leaves, whatever the treatment provided. In contrast, $S$. maritima leaves present a severe coloration from $400 \mu \mathrm{M}$ phe, reflecting a strong oxidative stress. Scale bars $=1.5 \mathrm{~mm}$.

Figure 5. GSTs phylogenetic reconstruction by Maximum Likelihood method based on the TVM (transversion) substitution model with a discrete Gamma distribution (4 categories). In total, $78 \mathrm{CDS}$ sequences were considered among A. thaliana, O. sativa, S. bicolor, Z. mays, $O$. thomaeum and Spartina. Tree is rooted by CDS sequences from the Omega GST gene family, and numbers at the nodes represent ultrafast bootstrap values from 10,000 replicates. Spartina contigs are displayed with black triangles, those we evaluate their expression by RT-qPCR are stained and belong to the Tau family. Corresponding sequences IDs: GRMZM- for Z. mays; Sobic- for S. bicolor; Oropecium- for O. thomaeum. Scale bar indicates 0.1 residue substitution per site.

Figure 6. Relative expression levels of five candidates contigs (GST homologs) estimated by RT-qPCR on three biological and technical replicates for each Spartina species in control conditions and under phenanthrene induced stress $(0$ and $400 \mu \mathrm{M})$. Values were calculated with the $2^{-\Delta \mathrm{Cp}}$ method; bars correspond to standard errors. **: p.value $<0.01$. 
Figure 1. 
A

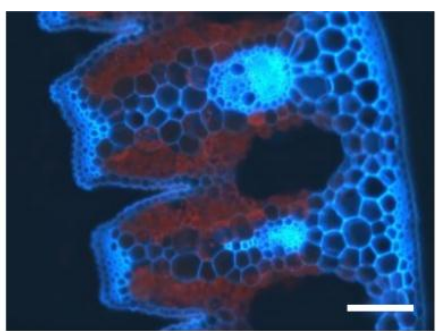

S. anglica

B
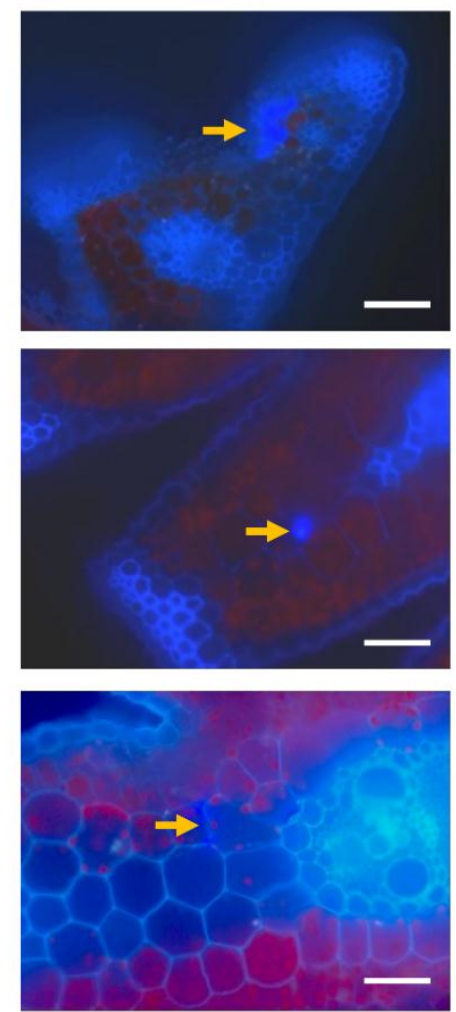

S. anglica

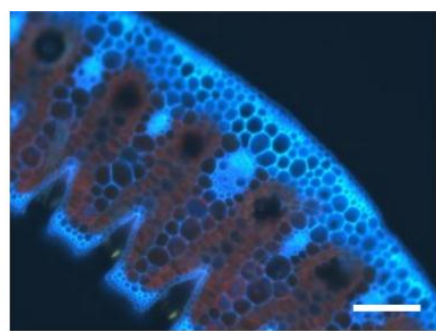

S. alterniflora
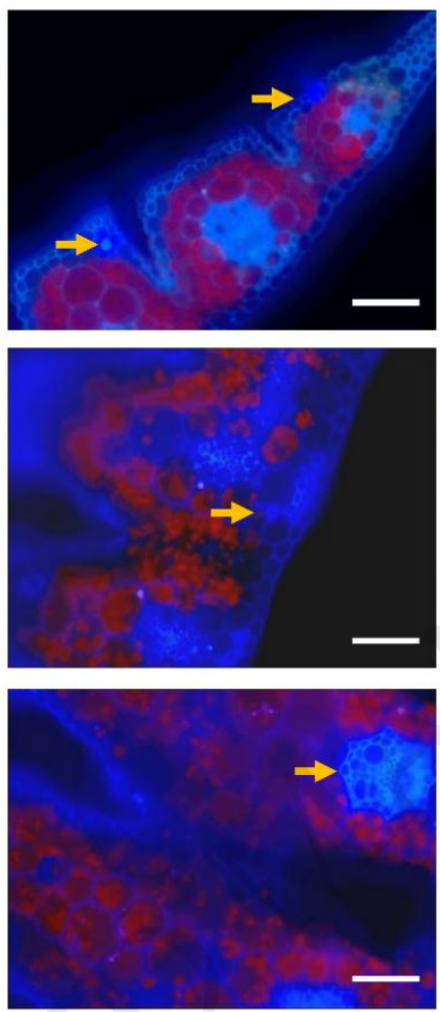

S. alterniflora

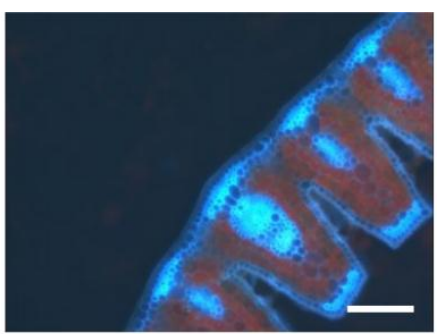

S. maritima
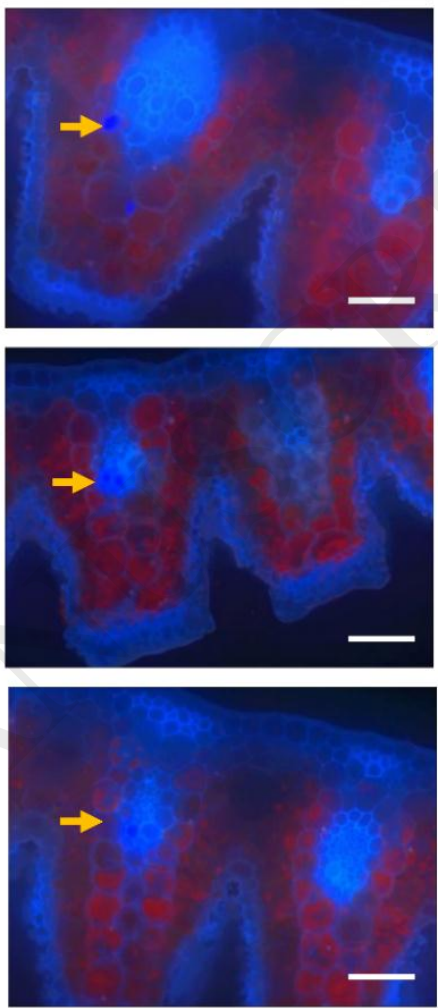

S. maritima

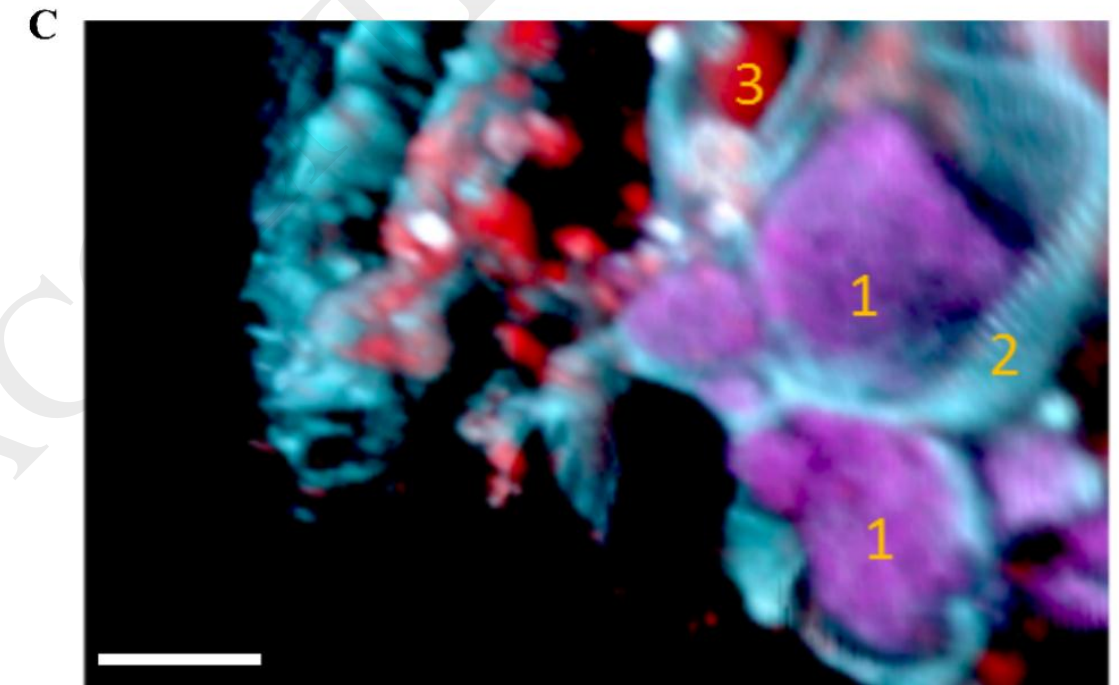

Figure 2. 


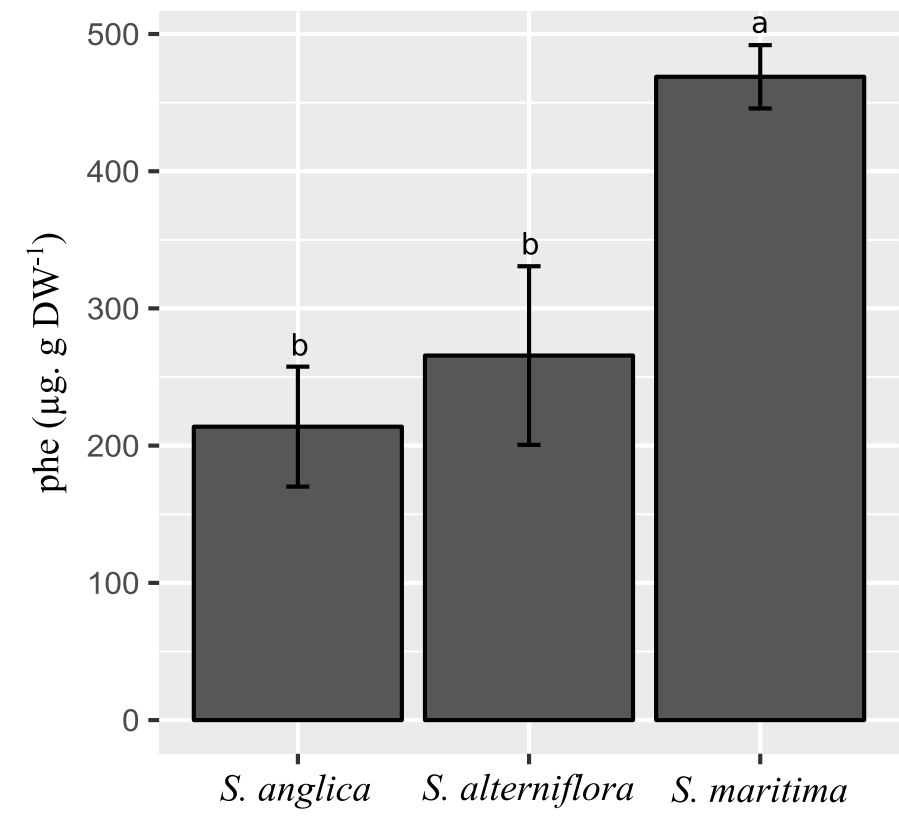

Figure 3.

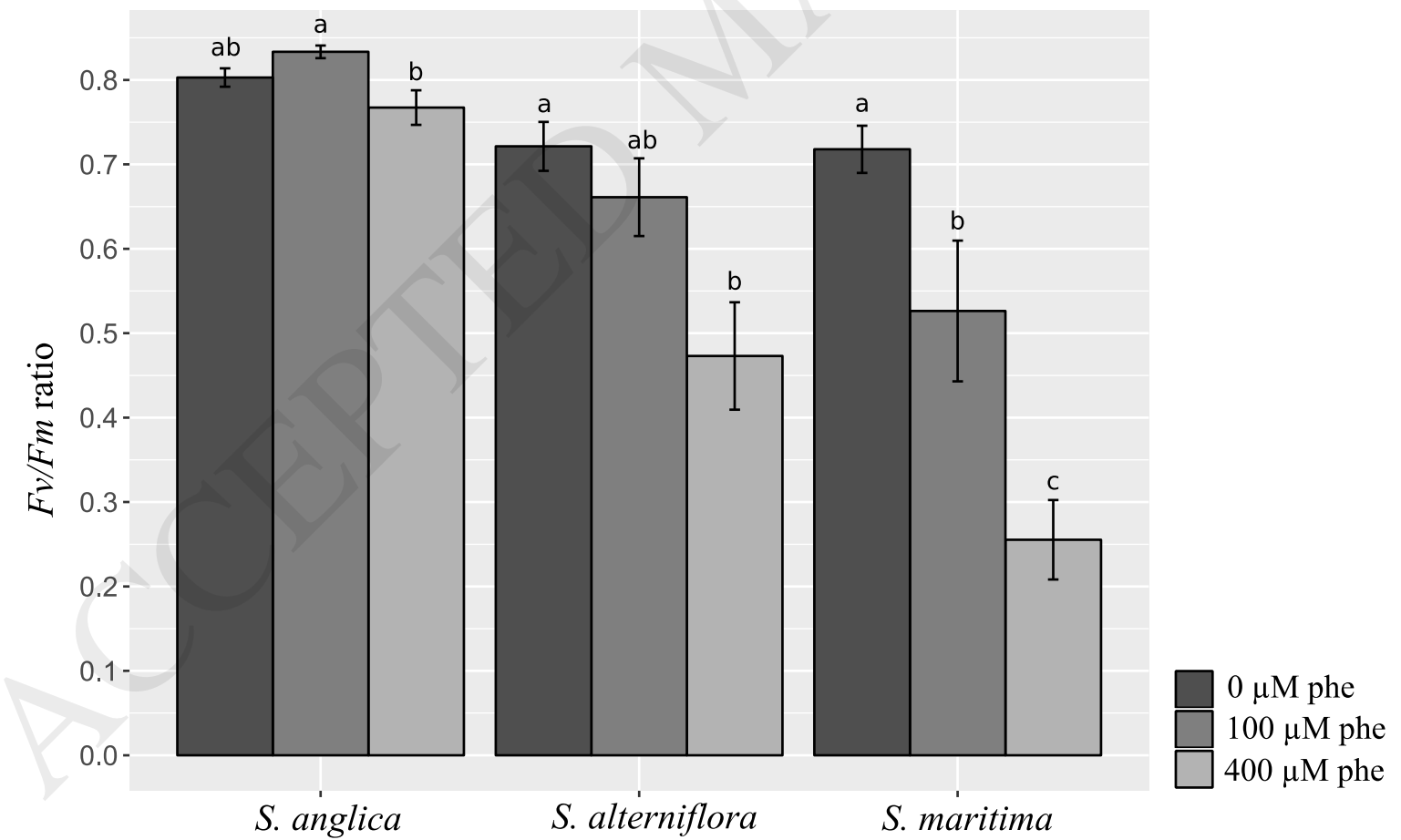

Figure 4. 

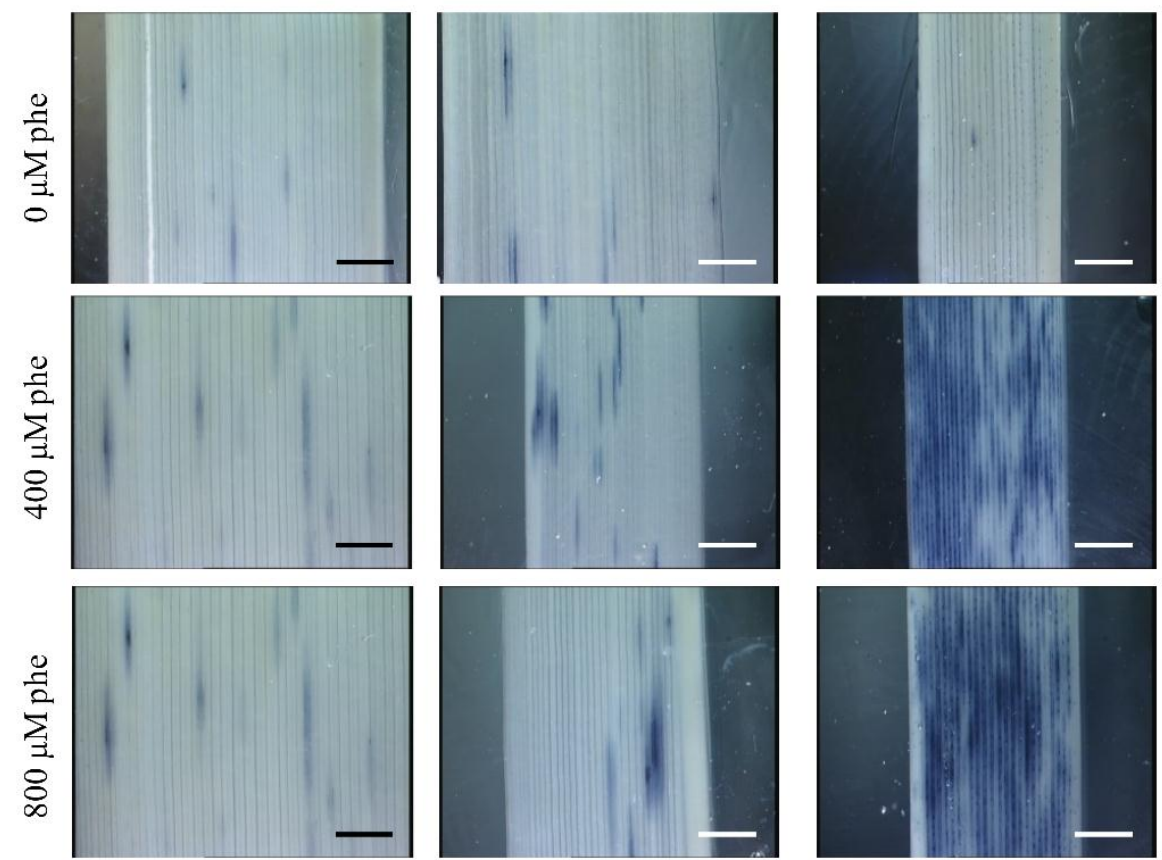

S. anglica

S. alterniflora

S. maritima

Figure 5. 


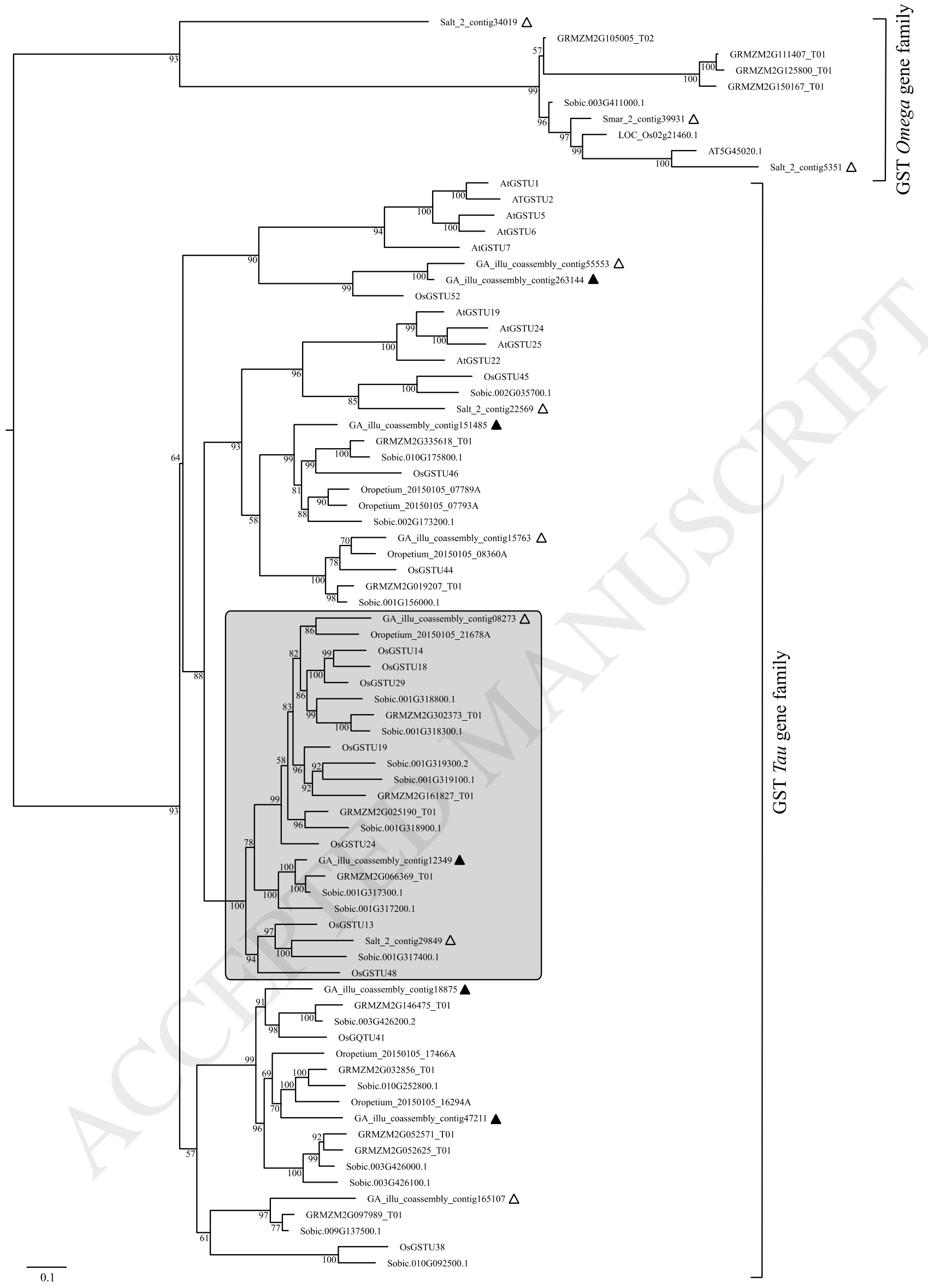

Figure 6. 
A Contig GA_47211

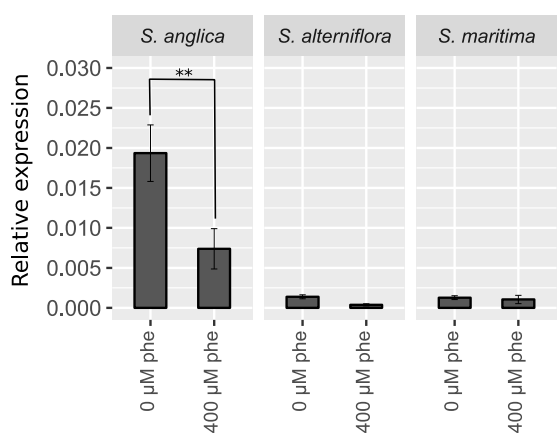

C Contig GA_12349

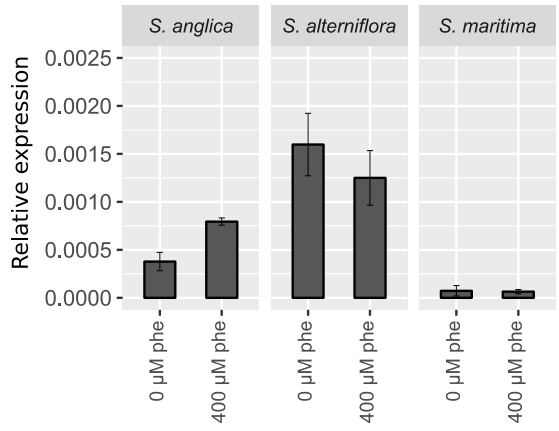

E Contig GA_263144

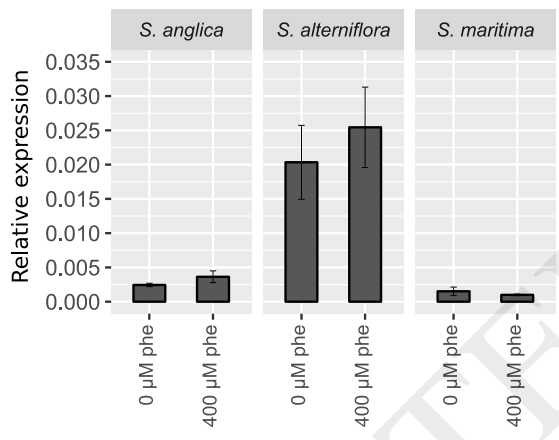

B Contig GA_151485

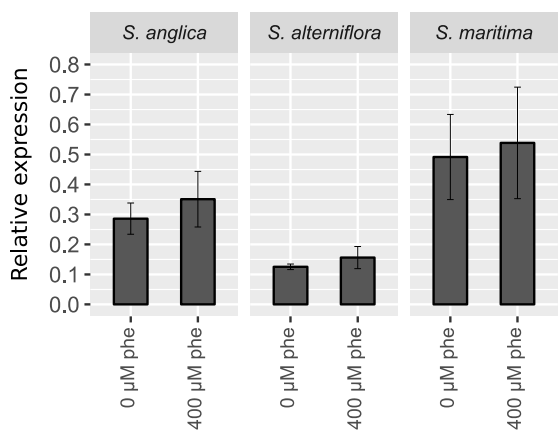

D Contig GA_15763

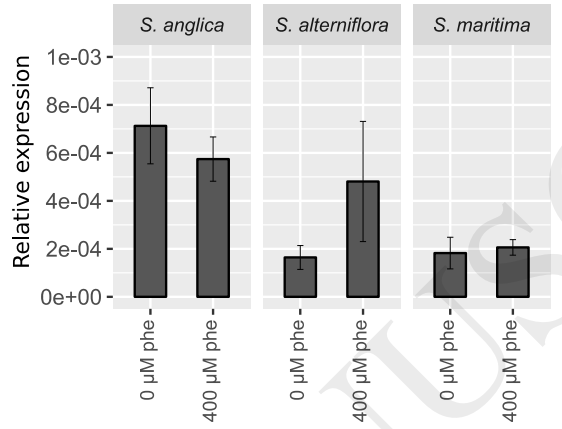


Table 1. Primers designed and validated for the six GSTs homologs in Spartina used for the RT-qPCR. Related A. thaliana genes and supplemental comments are provided.

\begin{tabular}{|l|l|l|l|l|l|}
\hline $\mathbf{N}^{\circ}$ & Spartina contigs & A. thaliana related homologs & Forward primer $(\mathbf{5}, \boldsymbol{\rightarrow} \mathbf{3}$ ) & Reverse primer $\left(\mathbf{5}^{\prime} \rightarrow \mathbf{3}^{\prime}\right)$ & Supplemental comments \\
\hline 1 & GA_47211 & AtGSTU6, AtGSTU7, AtGSTU8 & GATTCTGGGCCCACTTCTTC & TCGTCTCTTCCGTGAACTCC & - \\
\hline 2 & GA_151485 & AtGSTU25, AtGSTU22, AtGSTU19 & GACGTAGTCTGCCCAGAAGC & AGAAGATCCCAGTGCTGCTC & - \\
\hline 3 & GA_12349 & AtGSTU14 & GAGGTCCTGCTCGACGTACT & GAGGCAACTGCTAGAGCCTG & - \\
\hline 4 & GA_02873 & AtGSTU5 & GAGTTTGGTGGCGCTAAATG & AGACTTGCCCTCAGCTTCAA & Deleted because of primer dimers \\
\hline 5 & GA_15763 & AtGSTU24 & GACGAGGGACTCGCACAC & GTGGCGTACGAGGAGAAATC & - \\
\hline 6 & GA_263144 & AtGSTU1 & AGCTGCGGCATCTGGT & TGTTCTTGAGATCCTCCTCGAT & - \\
\hline
\end{tabular}

Table 1. 